\title{
Hepatic Encephalopathy
}

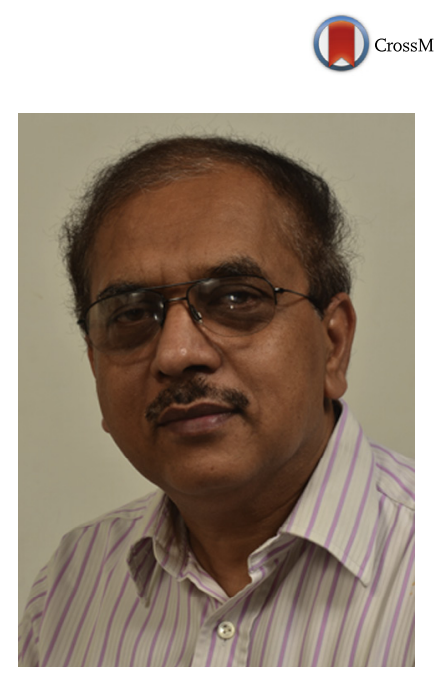

\author{
Radha K. Dhiman, MD, DM, FAMS, FACG, \\ Editor-in-Chief \\ Department of Hepatology, Postgraduate Institute of Medical Education \& Research, \\ Chandigarh 160012, India \\ E-mail: rkpsdhiman@hotmail.com
}

This issue of the Journal contains several articles on hepatic encephalopathy (HE) that represent a tremendous effort to pay tribute to Joan (Juan) Cordoba, who was a dear colleague and friend of those involved in HE research. For this purpose, International Society for HE and Nitrogen Metabolism (ISHEN) and Indian National Association for the Study of the Liver (INASL) decided to bring a supplement issue of "Journal of Clinical and Experimental Hepatology" (JCEH) on HE in order to honor Juan's contribution to HE. This supplement issue of JCEH has contributions from the experts in this field. Much emphasis was given to include articles on all aspects of HE. Emphasis was also given to include the latest developments and research in this field.

The history of HE is briefly but comprehensively reviewed. The historical review spans from the Hippocratic era to the present time including the American Association for the Study of Liver Diseases (AASLD) - the European Association for the Study of the Liver (EASL) consensus on this subject. There are three articles on different aspects of pathogenesis of HE. These describe in detail the synergistic relationship between ammonia and systemic inflammation, including the role of sepsis and inflammatory cytokines in the development of HE, ammonia induced microglial activation and local inflammatory responses in the brain resulting in neuroinflammation, and the role of altered structure and function of gut microbiota (dysbiosis) in the development of HE, i.e. the gut-brain axis. The

Abbreviations: AASLD: American Association for the Study of Liver Diseases; EASL: European Association for the Study of the Liver; HE: hepatic encephalopathy; HRQOL: health-related quality of life; INASL: Indian National Association for the Study of the Liver; ISHEN: International Society for HE and Nitrogen Metabolism; PEG: Polyethylene glycol http://dx.doi.org/10.1016/j.jceh.2015.02.001 better understanding of pathogenesis of HE is crucial to pave the way for development of novel therapeutic agents for the treatment of patients with HE.

Among the most outstanding achievements in recent times has been reaching a consensus and publishing AASLD-EASL guidelines on management of HE in chronic liver disease. A multiaxial classification has been developed over the recent years, which is based on underlying etiology, clinical severity, time course and presence or absence of precipitating factors. The article on definitions and nomenclature describes the same in a simple and practical manner. The new classification is expected to bring uniformity in the categorization of HE, improving patient care and outcome and encouraging clinical research across centers all over the world. There are three interesting articles on minimal HE, which include diagnosis of minimal HE and its impact on health-related quality of life (HRQOL) and sleep. Patients with minimal HE have impairment in almost in all domains of HRQOL which improves with treatment. There is higher frequency of sleep disturbances in patients with cirrhosis with minimal HE which include both night-time sleep disturbance and excessive daytime sleepiness. The neurophysiology of HE including potential role of EEG, quantified EEG, and evoked potentials-both exogenous, endogenous and motor-have been described in such a manner that may be useful to the readers who are unfamiliar with neurophysiology. Juan himself contributed an article on magnetic resonance imaging and spectroscopy in HE. He described various magnetic resonance technology in a simplistic manner. These technologies could be the handy tools for the brain study of liver failure to elucidate the time-course of the pathology, which may be useful in obtaining an early diagnosis of cerebral complications. 
Treatment of both covert and overt HE is challenging. There is no consensus on managing covert HE, hence larger randomized trials with good clinical measure of outcome are necessary to define the best therapeutic option for covert HE management. There is significant development in rationalizing the use of existing therapies such as lactulose and rifaximin both in the treatment of an acute episode of overt $\mathrm{HE}$ as well as in the secondary prophylaxis of HE. Role of many agents like L-ornithine L-aspartate, Lornithine phenyl acetate, probiotics, zinc, sodium benzoate, glycerol phenylbutyrate (HPN-100), and polyethylene glycol 3350-electrolyte solution (PEG) in the management of overt HE needs to be defined further in prospective randomized control trial. Pathogenesis and management of HE in acute liver failure are most clearly and comprehensively presented.

Finally, this supplement on HE includes two very special topics-encephalopathy in Wilson disease and approach to clinical syndrome of jaundice and encephalopathy in tropics. In clinical practice in a patient presenting with Wilsonian cirrhosis neuropsychiatric symptoms, it is challenge to determine if symptoms are caused either by the metabolic consequences of liver failure or by copper toxicity. Precise clinical skills and awareness may help. A large number of patients present with jaundice and encephalopathy in tropical country like India, which may be caused by tropical infections such as malaria, dengue fever, leptospirosis, typhoid fever, etc. These conditions may mimic acute liver failure but may have subtle differences from acute liver failure. This review comprehensively summarizes an approach to clinical syndrome of jaundice and encephalopathy in tropics.

The Editors are indebted to the authors for their exceptional contributions to the supplement issue of "Journal of Clinical and Experimental Hepatology". 